\title{
Basic and Acidic Fibroblast Growth Factors Have Trophic Effects on Neurons from Multiple CNS Regions
}

\author{
Patricia Ann Walicke ${ }^{1,2}$ \\ 'The Developmental Neurobiology Laboratory and The Clayton Foundation for Research-California Division, The Salk \\ Institute for Biological Studies, and 2Department of Neuroscience, University of California, San Diego, California 92093
}

\begin{abstract}
Basic fibroblast growth factor (bFGF) supports the survival of neurons from many regions of the E18 fetal rat brain. Survival was significantly increased for neurons derived from the hippocampus, entorhinal cortex (EC), frontal cortex, parietal cortex (PC), occipital cortex, striatum, septum, and thalamus, but not from the subiculum (Sb). The proportion of neurons rescued by bFGF varied among brain regions, suggesting the existence of subpopulations of responsive neurons. Like hippocampal neurons, neurons from the EC and $P C$ required about $1 \mathrm{pM}$ bFGF $(10-20 \mathrm{pg} / \mathrm{mi})$ for halfmaximal response; striatal neurons, in contrast, required about 3 pM bFGF. Neurite outgrowth after $24 \mathrm{hr}$ exposure was significantly increased for neurons from the hippocampus, EC, and PC, while striatal neurons had only a marginal response. Although bFGF stimulated some astrocytic proliferation in the cultures, glial contamination was maintained at $2 \%$ or less. Acidic FGF (aFGF) supported smaller numbers of neurons from each region, although it significantly increased survival of neurons from hippocampus, EC, PC, striatum, and $\mathrm{Sb}$. The concentration required for half-maximal survival was around $100-300 \mathrm{pm}(2-5 \mathrm{ng} / \mathrm{ml})$. It appears that bFGF and aFGF are potent trophic factors for many populations of CNS neurons and could potentially play a significant role in nervous system development.
\end{abstract}

The survival and growth of neurons throughout the CNS is presumed to be regulated by the availability of appropriate trophic factors. For the vast majority of neuronal populations, however, the molecular identity of these factors is unknown. Nerve growth factor (NGF), the one fully established neurotrophic factor, appears to address only a few populations of CNS neurons, specifically the cholinergic neurons of the septum, nucleus basilis, and striatum (Seiler and Schwab, 1984; Mobley et al., 1985, 1986; Hefti, 1986; Large et al., 1986; Taniuchi et al., 1986; Williams et al., 1986). Although other populations of NGF-responsive neurons will likely be identified (Large et al., 1986; Taniuchi et al., 1986; Williams et al., 1986), it appears

\footnotetext{
Received Aug. 24, 1987; revised Nov. 13, 1987; accepted Nov. 13, 1987.

I would like to thank Dr. Andrew Baird for his generous gift of bFGF and aFGF; Dr. David Amaral for providing insight into fetal brain anatomy and the generous loan of his digitizing equipment; Miss Cassandra Harrison for excellent technical assistance; Mr. Kris Trulock for assistance with the photography. These experiments were supported by the Clayton Foundation for Research-California Division, and were performed while P.W. was in receipt of a Teacher Investigator Development Award (1 K07 NS 00948-02).

Correspondence should be addressed to Patricia Ann Walicke, MD, PhD, Dept. of Neuroscience, M-008, University of Calitornia, San Diego, CA 92093.

Copyright (C) 1988 Society for Neuroscience $0270-6474 / 88 / 072618-10 \$ 02.00 / 0$
}

that NGF is unlikely to be a primary neurotrophic factor for the majority of CNS neurons.

Recently, basic fibroblast growth factor (bFGF) has been demonstrated to increase the survival and growth of neurons in cultures derived from the hippocampus and whole cortex (Morrison et al., 1986; Walicke et al., 1986a; Walicke and Baird, 1987a). Further studies with hippocampal neurons have demonstrated that bFGF can support neuronal survival in cultures lacking any astrocytes detectable by staining with antisera for glial fibrillary acidic protein (GFAP) (Walicke and Baird, 1987b). Furthermore, ${ }^{125}$ I-bFGF binds to and is internalized by hippocampal neurons (Walicke and Baird, 1987a). Together these observations strongly suggest that bFGF truly has neurotrophic effects on this population of CNS neurons.

bFGF is found in adult brain tissue (Esch et al., 1985a; Baird et al., 1986; Gospodarowicz et al., 1986), although its regional and cellular distribution remains to be determined. In these studies, its possible role as a neurotrophic factor in other portions of the CNS is addressed by testing its effects on neurons from various regions of fetal rat brain in vitro. These studies extend the previous demonstrations that cortical neurons respond to bFGF (Morrison et al., 1986; Walicke and Baird, 1987a) by comparing neurons from different cortical regions to determine areas containing responsive neurons.

\section{Materials and Methods}

bFGF and acidic fibroblast growth factor (aFGF) were the generous gifts of Dr. Andrew Baird. bFGF was prepared from bovine pituitary by sequential steps of ammonium sulfate precipitation, CM-Sephadex chromatography, and hcparin-Scpharosc affinity chromatography as previously described (Esch et al., 1985a). Some experiments utilized bFGF after the heparin-Sepharose chromatography, which was about $90 \%$ homogenous. Other experiments used bFGF that had been further purified by a terminal step of high-performance liquid chromatography (HPLC) on a Mono S column. Results with the 2 preparations did not differ detectably and have been pooled in the results. aFGF was prepared from bovine brain by essentially the same series of steps, which always included a terminal purification on reverse-phase HPLC (Esch et al., 1985b).

All neuronal cultures were prepared from 18-d fetal Sprague-Dawley rats. The dissection scheme was in part derived from others (Banker and Cowan, 1979; Barbin et al., 1984), and for clarity is illustrated in Figure 1. The parahippocampal gyrus presents a major landmark in the E18 brain, allowing reliable definition of the hippocampus. Only subtotal portions of the major cortical regions-frontal, parietal, and occipital-were employed to allow clear separation of their neuronal populations. There are no clear boundaries for the entorhinal cortex (EC), so these cultures could be contaminated with some neurons from the adjoining hippocampus, occipital cortex, temporal cortex, and amygdala. The region designated subiculum (Sb) contains portions of retrosplenial cingulate cortex and possibly some occipital cortex. Thalamic 
cultures (Th) were derived only from tissue comprising the anterior dorsal quadrant of the thalamus (not illustrated). The septal region (Se) consisted of the collection of midline basal forebrain structures, in addition to the septal nuclei themselves. Since only a relatively small portion of the basal ganglion complex was employed, the cultures designated striatum $(\mathrm{St})$ should contain neurons only from that region.

Tissues from 5-15 fetal rats werc pooled in Hank's balanced salt solution (HBSS) with $20 \mathrm{~mm}$ HEPES, pH 7.4, and dissociated by incubation for 5-15 min (depending on tissue thickness) at $37^{\circ} \mathrm{C}$ in $0.25 \%$ trypsin in $\mathrm{Ca}^{2+} / \mathrm{Mg}^{2+}$-free HBSS. The protease was inactivated by subsequent rinsing with $60 \mu \mathrm{M}$ phenylmethylsulfonyl fluoride (PMSF) in $\mathrm{Ca}^{2+} / \mathrm{Mg}^{2^{+}}$-free HBSS. Dissociation was completed by trituration through a silane-coated Pasteur pipette. Viable cells were determined by trypan blue exclusion. Sb neurons were grown at a density of 9,000 cells $/ \mathrm{cm}^{2}$ and all other neurons at 18,000 cells $/ \mathrm{cm}^{2}$ in a modification of the $\mathrm{N} 2$ serum-free medium formulation of Bottenstein and Sato (1979), as previously described (Walicke et al., 1986a; Walicke and Baird, 1987b).

For survival studies, neurons were grown on plastic or polyornithinecoated dishes for $6 \mathrm{~d}$. The cultures were fixed with $4 \%$ glutaraldehyde, and process-bearing cells were counted in $5-10 \%$ of the culture area under phase microscopy. For process outgrowth studies, neurons werc grown on culture plates coated with polyornithine and laminin (Walicke et al., 1986a) in medium supplemented with $20 \mu \mathrm{g} / \mathrm{ml}$ high-density lipoprotein (Sigma) for $24 \mathrm{hr}$ and then fixed with glutaraldehyde. The central portions of 4 cultures were photographed at $20 \times$ under phase optics. The photos were enlarged to $8 \times 10$ and the longest process of each cell was measured on a Bausch and Lomb HiPad digitizer (Walicke et al., 1986a).

Immunohistochemistry for GFAP was performed as previously described (Walicke and Baird, 1987b). The entire culture area was examined and counted for determination of the astrocyte number.

\section{Results}

Basic FGF increases survival of neurons from many regions of the CNS

Nine brain regions were included in the initial survey: hippocampus $(\mathrm{H})$, entorhinal cortex (EC), subiculum (Sb), frontal cortex $(\mathrm{FC})$, parietal cortex $(\mathrm{PC})$, occipital cortex $(\mathrm{OC})$, septal region $(\mathrm{Se})$, anterior thalamus (Th), and striatum (St). The effects of bFGF on the survival of neurons from these regions are presented in Figure 2. The upper half of Figure 2 indicates neuronal survival after $24 \mathrm{hr}$ in vitro in the presence and absence of bFGF $(1 \mathrm{ng} / \mathrm{ml}$ ). Although there is some variability, about $40-60 \%$ of neurons from each region survived for $24 \mathrm{hr}$. Survival was not significantly altered by inclusion of bFGF for neurons from any region except the frontal cortex, where neuronal number was increased at a marginal level of significance $(p<0.05)$. The basal culture medium contained pyruvate, catalase, and elevated $\mathrm{K}^{+}$, all of which have been reported to enhance the initial survival of primary neurons in vitro (Lasher and Zagon, 1972; Selak et al., 1985; Walicke et al., 1986b).

The number of neurons surviving after $6 \mathrm{~d}$ in vitro is shown in the lower half of Figure 2. The presence of bFGF $(1 \mathrm{ng} / \mathrm{ml})$ significantly increased the survival of neurons from all of the regions examined, with the exception of the $\mathrm{Sb}$. It is apparent that larger numbers of neurons survived in the presence of $b F G F$ in tissue derived from cortical regions than in tissue from subcortical regions; however, survival in control cultures from subcortical regions was also lower. A more accurate assessment of the relative effect of $b F G F$ can be obtained from consideration of the proportion of neurons rescued in its presence. This was calculated as the difference in neuronal number between $6 \mathrm{~d}$ cultures with and without bFGF, normalized to the average number of neurons present at $24 \mathrm{hr}$ in vitro. The quantity calculated for each region appears below the bar graph in Figure 2.

Neurons from the hippocampus appeared to be most respon-
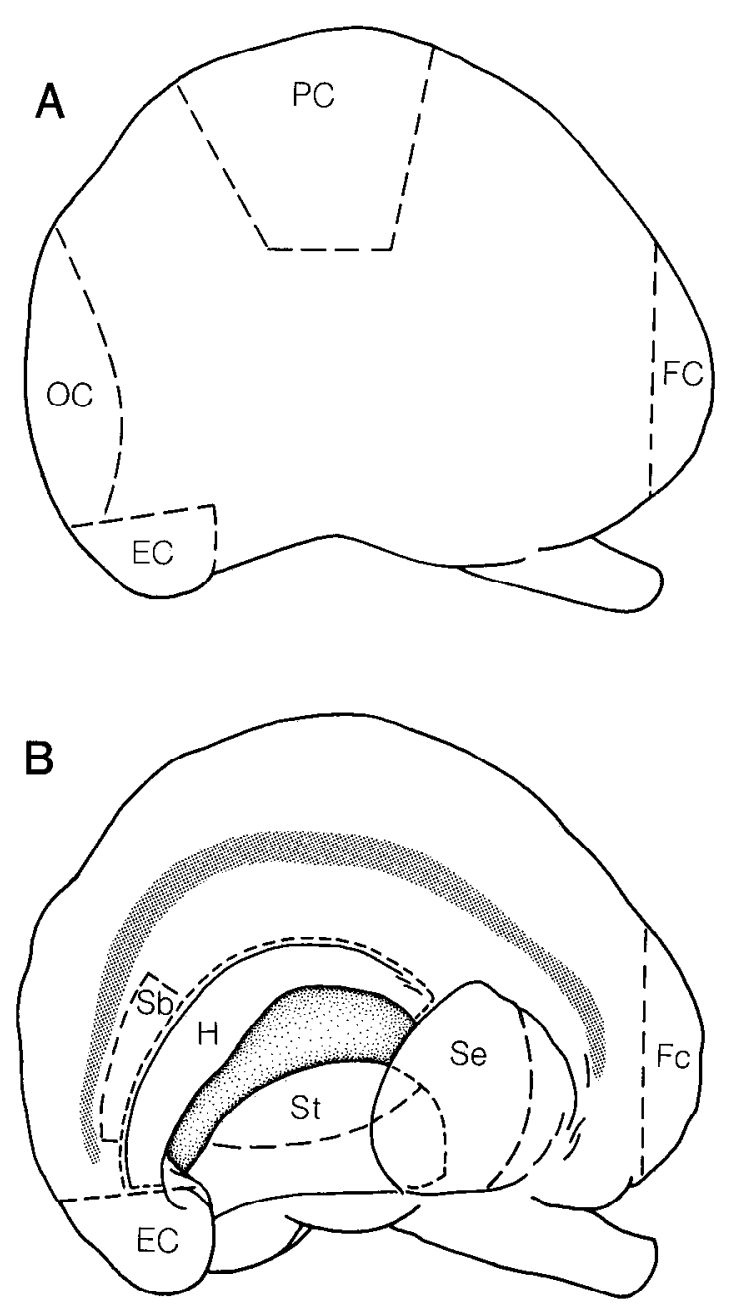

Figure 1. Illustration of the dissection methods. Diagrams of sagittal views of the E18 fetal rat brain after removal of the meninges, brain stem, and thalamus. $A$, Lateral view. $B$, Medial view. Dashed lines indicate cuts made. $H$, hippocampus; $E C$, entorhinal cortex; $F C$, frontal cortex; $P C$, parietal cortex; $O C$, occipital cortex; $S b$, subiculum; $S t$, striatum; Se, septum.

sive, with about $70 \%$ rescued by bFGF. Most of the cortical regions showed increases of $30-45 \%$, including the EC, OC, and FC, while the PC's was slightly lower at $21 \%$. The thalamus and striatum also showed increases in survival in this range, demonstrating that there was no real difference between cortical and subcortical regions. Even adjusting for the lower survival in the controls, neurons from the septal region showed relatively little response to bFGF, with only a $12.2 \%$ increase in survival. Again, the least responsive region was the $\mathrm{Sb}$, with only a $3 \%$ change in the presence of bFGF.

Because of variability in survival between experiments, these quantities must be interpreted with some caution. Several regions were selected for more quantitative assessment. Examples of cultures maintained for $6 \mathrm{~d}$ in the presence of bFGF from these regions-H, EC, $\mathrm{Sb}, \mathrm{PC}$, and $\mathrm{St}-$ are shown in Figure 3. The cultures from the first 4 regions are rather similar in appearance, containing predominantly pyramidal and stellateshaped neurons with an extensive process network. The striatal neurons were noticeably smaller and, even after $6 \mathrm{~d}$, had only short processes that did not form a complex nctwork. The vast majority of cells present in cultures from each brain region were 
Figure 2. Effects of bFGF on neuronal survival. Abbreviations, as in Figure 1; $T h$, thalamus. All cultures were grown on plastic plates, as described in Materials and Methods. bFGF was $1 \mathrm{ng} /$ ml. Top, Survival after $24 \mathrm{hr}$ in vitro. Bottom, Survival after $6 \mathrm{~d}$ in vitro. Bars are means \pm SEM for 6 duplicate cultures. Numbers in parentheses are percentages of neurons present after $24 \mathrm{hr}$ surviving to $6 \mathrm{~d}$ in the presence of bFGF. Numbers under the graph represent the percentage of neurons rescued by $b F G F$, calculated as in text. ${ }^{*} p<0.01$ versus control (ANOVA).

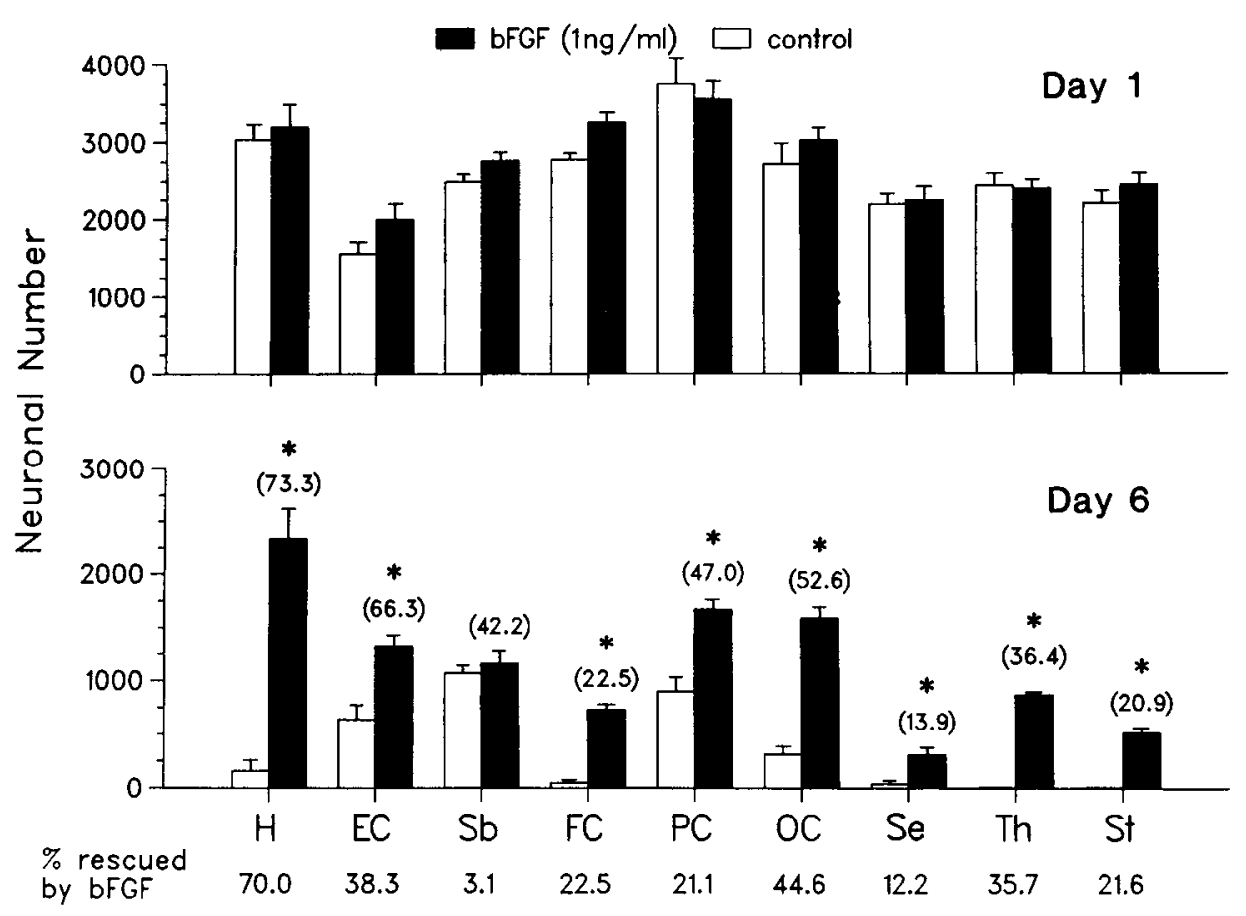

Under control conditions, there was a trend toward lowest survival in St cultures and highest survival in Sb cultures; however, because of variability between experiments, there were no significant differences. It should be noted that $\mathrm{Sb}$ neurons were plated at a lower density than the others because of their tendency to survive well under control conditions. In the presence of bFGF, hippocampal neurons survived best, followed by neurons from the EC and St. For all 3 regions, more than $50 \%$ of cells remained at the end of the assay period. Survival was somewhat lower for the $\mathrm{PC}$, but neurons from the $\mathrm{Sb}$ had the worst survival in the presence of $\mathrm{bFGF}$, differing significantly from the hippocampus ( $p<0.01$; ANOVA) and EC $(p<0.05$; ANOVA).

The proportion of neurons rescucd by inclusion of bFGF was greatest in the hippocampus, at about $60 \%$ of the population. Neurons from the EC and striatum each showed increases of about $38 \%$. Only $24 \%$ of $\mathrm{PC}$ neurons were rescued by bFGF, hr cultures with and without bFGF was used.

Table 1. Comparison of different neuronal responses to bFGF

\begin{tabular}{llllc} 
& $\begin{array}{l}\text { Concentration } \\
\text { of bFGF } \\
(\mathrm{pg} / \mathrm{ml})\end{array}$ & $\begin{array}{l}\text { Survival } \\
\text { control } \\
(\%)\end{array}$ & $\begin{array}{l}\text { Survival } \\
\text { of bFGF } \\
(\%)\end{array}$ & $\begin{array}{l}\text { Survival } \\
\text { increased } \\
\text { by bFGF } \\
(\%)\end{array}$ \\
\hline Hippocampus & $13.3 \pm 1.7$ & $14.9 \pm 4.8$ & $69.3 \pm 7.6$ & $59.4 \pm 9.5$ \\
Entorhinal cortex & $8.3 \pm 2.4$ & $22.1 \pm 7.2$ & $56.5 \pm 9.2$ & $38.9 \pm 9.3$ \\
Subiculum & N.D. & $23.8 \pm 6.4$ & $23.0 \pm 5.4^{*}$ & $2.6 \pm 1.0^{*}$ \\
Parietal cortex & $18.8 \pm 6.6$ & $18.2 \pm 5.8$ & $40.2 \pm 5.0^{* *}$ & $24.3 \pm 3.9^{*}$ \\
Striatum & $48.8 \pm 5.2^{*}$ & $9.4 \pm 3.5$ & $50.5 \pm 6.7$ & $38.4 \pm 5.4$
\end{tabular}

Effects of bFGF on neuronal survival. Neuronal survival was determined as described in Materials and Methods. The concentration of bFGF required for half-maximal survival represents the mean $\pm S E M$ for determinations in 4 experiments, each using 3-4 duplicate cultures. Percentage survival figures represent the mean \pm SEM of determinations in 8 individual experiments using 4-6 duplicate cultures each. The concentrations of bFGF was $1 \mathrm{ng} / \mathrm{ml}$.

N.D., not determinable.

${ }^{*} p<0.01$ versus hippocampus (ANOVA).

** $p<0.05$ versus hippocampus (ANOVA). 

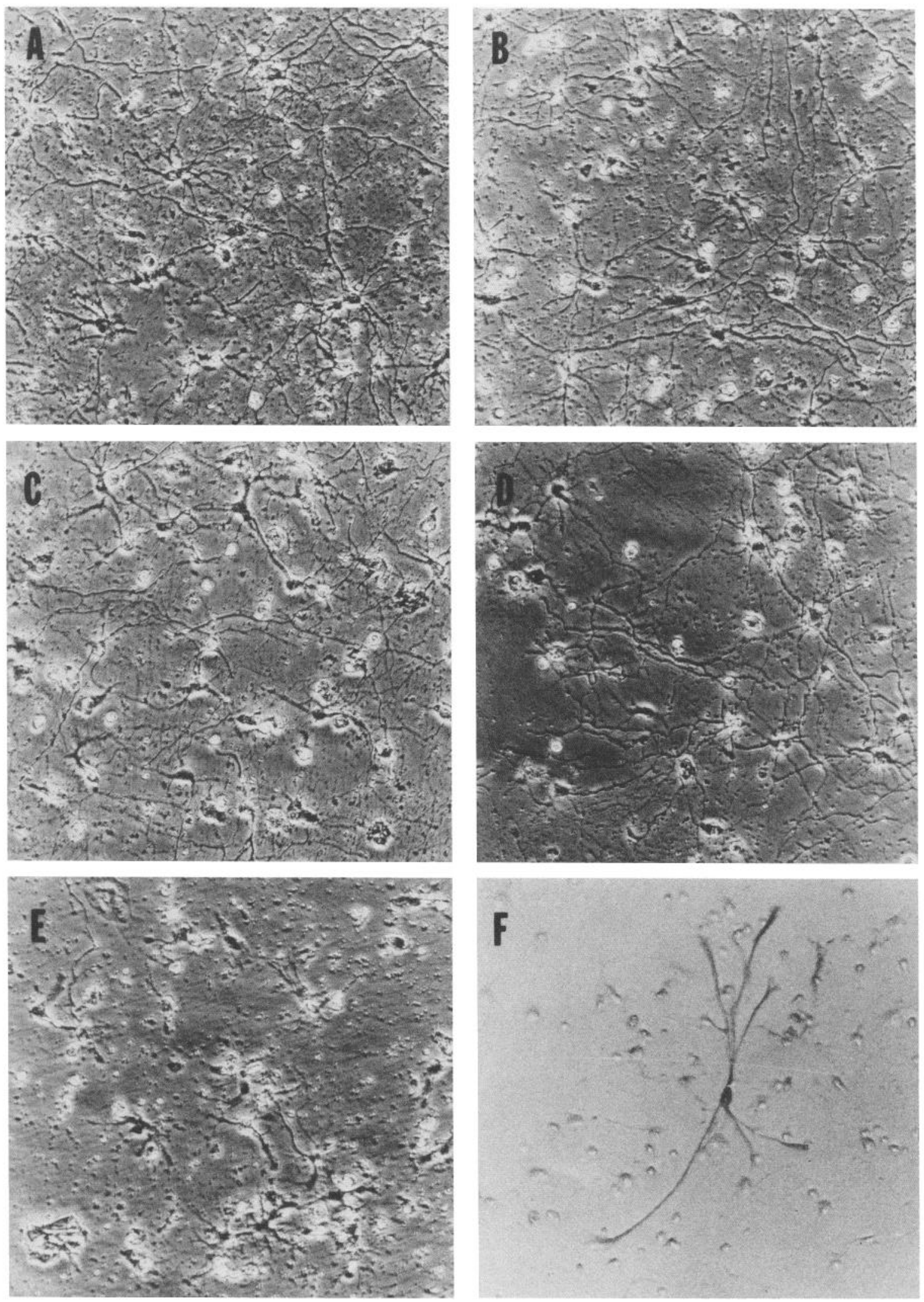

Figure 3. Photographs illustrating the appearance of 6-d-old cultures grown in the presence of $1 \mathrm{ng} / \mathrm{ml} \mathrm{bFGF}$ for neurons from $(A) \mathrm{H}$; $(B) \mathrm{EC}$; $(C) \mathrm{Sb} ;(D) \mathrm{PC} ;(E) \mathrm{St}$. $F$, An astrocyte stained with antisera to GFAP in a 6-d-old culture of hippocampal neurons grown with $1 \mathrm{ng} / \mathrm{ml}$ bFGF. $A-$ $E$, Phase-microscopic views. $F$, Transmitted light. Magnification, $200 \times$. 

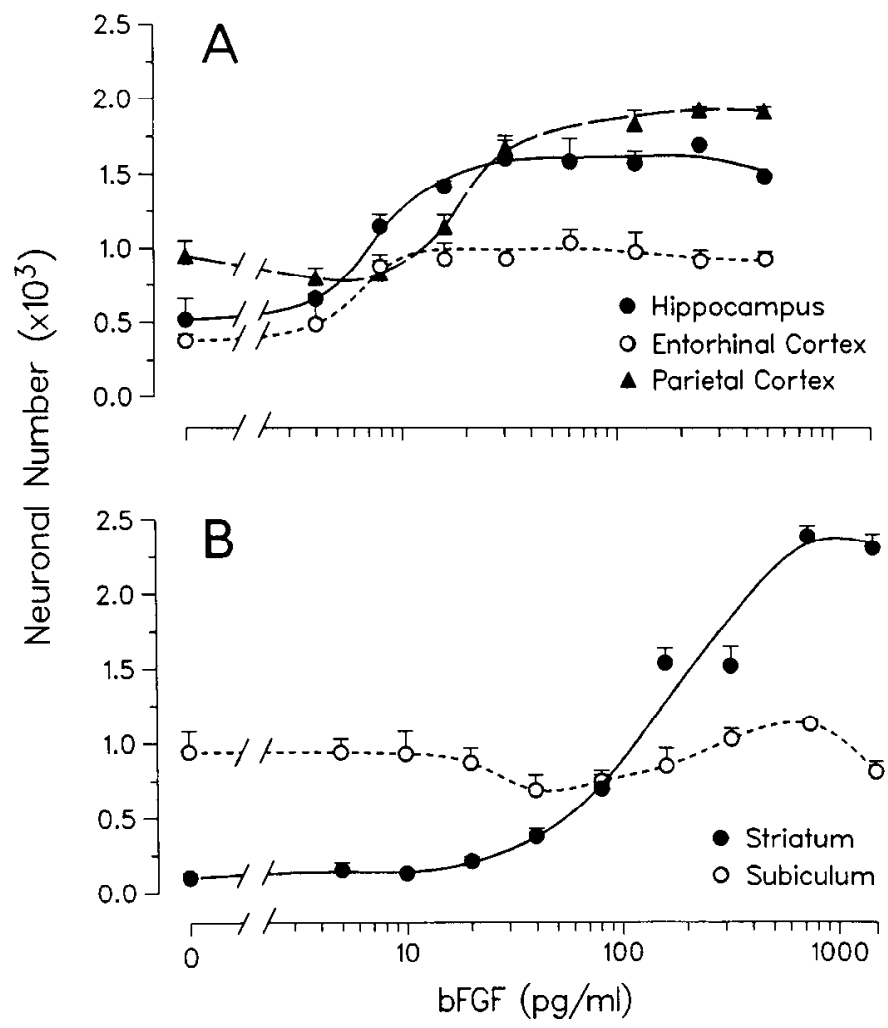

Figure 4. Titration curves of bFGF with various neuronal types. $A$, Neurons from hippocampus, EC, and PC, as indicated. $B$, Neurons from $\mathrm{Sb}$ and $\mathrm{St}$, as indicated. Points are mean $\pm \mathrm{SEM}$ for 4 duplicate cultures.

which differed significantly from observations with the hippocampus ( $p<0.01$; ANOVA). The $\mathrm{Sb}$ appeared to have little response to bFGF, with less than $3 \%$ of cells rescued, which differed significantly from the hippocampus, EC, and striatum $(p<0.01$; ANOVA).

The minimal concentration of bFGF required to support survival was determined for neurons from each of these regions by performing titration curves of bFGF. The results of determinations in 4 independent experiments were averaged in Table 1. Examples are illustrated in Figure 4. Neurons from the hippocampus, EC, and PC produced similar titration curves, showing cell number rising rapidly as a function of bFGF concentration to reach a single plateau of survival for that population. The quantity of bFGF calculated to support half-maximal neuronal survival ranged between about 5 and $25 \mathrm{pg} / \mathrm{ml}(0.3-1.5$ pM), consistent with previous determinations using hippocampal neurons (Walicke et al., 1986a). Striatal neurons required a slightly but significantly higher concentration of bFGF for survival, about $50 \mathrm{pg} / \mathrm{ml}(3 \mathrm{pM})(p<0.01$, as compared to hippocampus, EC, and $\mathrm{PC}$ ). Titration curves performed with neurons from the $\mathrm{Sb}$ produced a straight line (Fig. $4 B$ ). Concentrations in excess of $3 \mathrm{ng} / \mathrm{ml}$ were not examined.

In addition to its effects on neuronal survival, bFGF stimulates astrocytes to proliferate and to adopt a fibrillar morphology (Morrison and de Vellis, 1981; Pettmann et al., 1985; Walicke and Baird, 1987b). Since glial cells have been demonstrated to support neuronal survival in vitro (Banker, 1980; Selak et al., 1985; Gloor et al., 1986), it is important to determine the proportion of neurons and astrocytes present in these various cultures. Therefore, cultures were stained with antisera to GFAP for identification of astrocytes; an example of a large fibrillar astrocyte from a hippocampal culture is shown in Figure $3 F$.

\begin{tabular}{lllll}
\hline Table 2. Astrocytic contents of neuronal cultures & \\
& & & \\
& & & \multicolumn{1}{l}{$\begin{array}{l}\text { Glial } \\
\text { cells }\end{array}$} \\
& & Neurons & Astrocytes & \multicolumn{1}{l}{$(\%)$} \\
\hline $\mathrm{H} \quad$ Control & $1220 \pm 70$ & $8.4 \pm 1.2$ & 0.7 \\
& + bFGF & $2960 \pm 80$ & $55.1 \pm 2.3$ & 1.9 \\
$\mathrm{EC}$ & Control & $1070 \pm 120$ & $1.0 \pm 0.4$ & 0.1 \\
& + bFGF & $2130 \pm 120$ & $5.1 \pm 1.2$ & 0.2 \\
$\mathrm{Sb}$ & Control & $1400 \pm 90$ & $1.5 \pm 0.4$ & 0.1 \\
& + bFGF & $1520 \pm 60$ & $5.0 \pm 1.2$ & 0.3 \\
$\mathrm{PC}$ & Control & $930 \pm 80$ & $1.0 \pm 0.6$ & 0.1 \\
& + bFGF & $2220 \pm 90$ & $4.6 \pm 1.4$ & 0.2 \\
$\mathrm{St}$ & Control & $500 \pm 60$ & $0.8 \pm 0.6$ & 0.2 \\
& + bFGF & $1470 \pm 160$ & $5.4 \pm 2.1$ & 0.4 \\
\end{tabular}

Glial contents of neuronal cultures. Cultures from the indicated regions were grown for $6 \mathrm{~d}$ in the presence or absence of $1 \mathrm{ng} / \mathrm{ml} \mathrm{bFGF}$. Astrocytes were determined by staining for GFAP and examining the entire culture; neurons were counted in $5 \%$ of the culture area. Determinations are mean \pm SEM for 8 duplicate cultures.

The proportion of astrocytes present in cultures maintained for $6 \mathrm{~d}$ in control medium or the presence of bFGF is summarized in Table 2. Despite the use of serum-free medium, bFGF stimulated some proliferation of astrocytes in cultures from each brain region. The hippocampal cultures contained the highest number of glial cells, averaging 55 astrocytes/culture with bFGF. However, astrocytes still represented less than $2 \%$ of the total cells present. Even in the presence of bFGF, the other regions surveyed contained only around $1.0-1.0 \%$ astrocytes. Previous analyses of hippocampal cultures have demonstrated no correlation between astrocyte and neuronal number in cultures containing up to $1.0 \%$ astrocytes, suggesting that the rare glial cells have no significant influence on survival of the neuronal population (Walickc and Baird, 1987b). This conclusion likely applies as well to these other brain regions.

\section{Neurite outgrowth stimulated by $b F G F$}

The survival studies were performed using a minimal, chemically defined medium to avoid inadvertent inclusion of growthpromoting or inhibiting factors in complex additives such as serum. After $6 \mathrm{~d}$, neurons from all regions studied, with the exception of the striatum, showed much more extensive neuritic networks in the presence of bFGF. However, this difference could have reflected only the greater number of healthy surviving neurons rather than a specific effect of bFGF on process elongation. Therefore, neurite outgrowth was examined after 24 $\mathrm{h}$ in vitro, before there were significant differences in neuronal survival in the presence of bFGF.

Neurite outgrowth is a complex cellular function that appears to be regulated by multiple influences beyond the availability of a trophic factor. Previous studies with hippocampal neurons have demonstrated that a small but significant response to a trophic factor can be overlooked if culture conditions are not optimal for process extension (P. A. Walicke, unpublished observations). A variety of conditions were surveyed with these different neuronal populations, including different growth surfaces-plastic, polyornithine, polyornithine-heparin, and polyornithine-laminin - and medium supplements of triiodothyronine, hydrocortisone, and high-density lipoproteins (HDL). It appeared that addition of HDL increased early neurite outgrowth quite effectively (Walicke, 1987), probably by providing a source of lipids complexed with apolipoprotein E (Ignatius et 

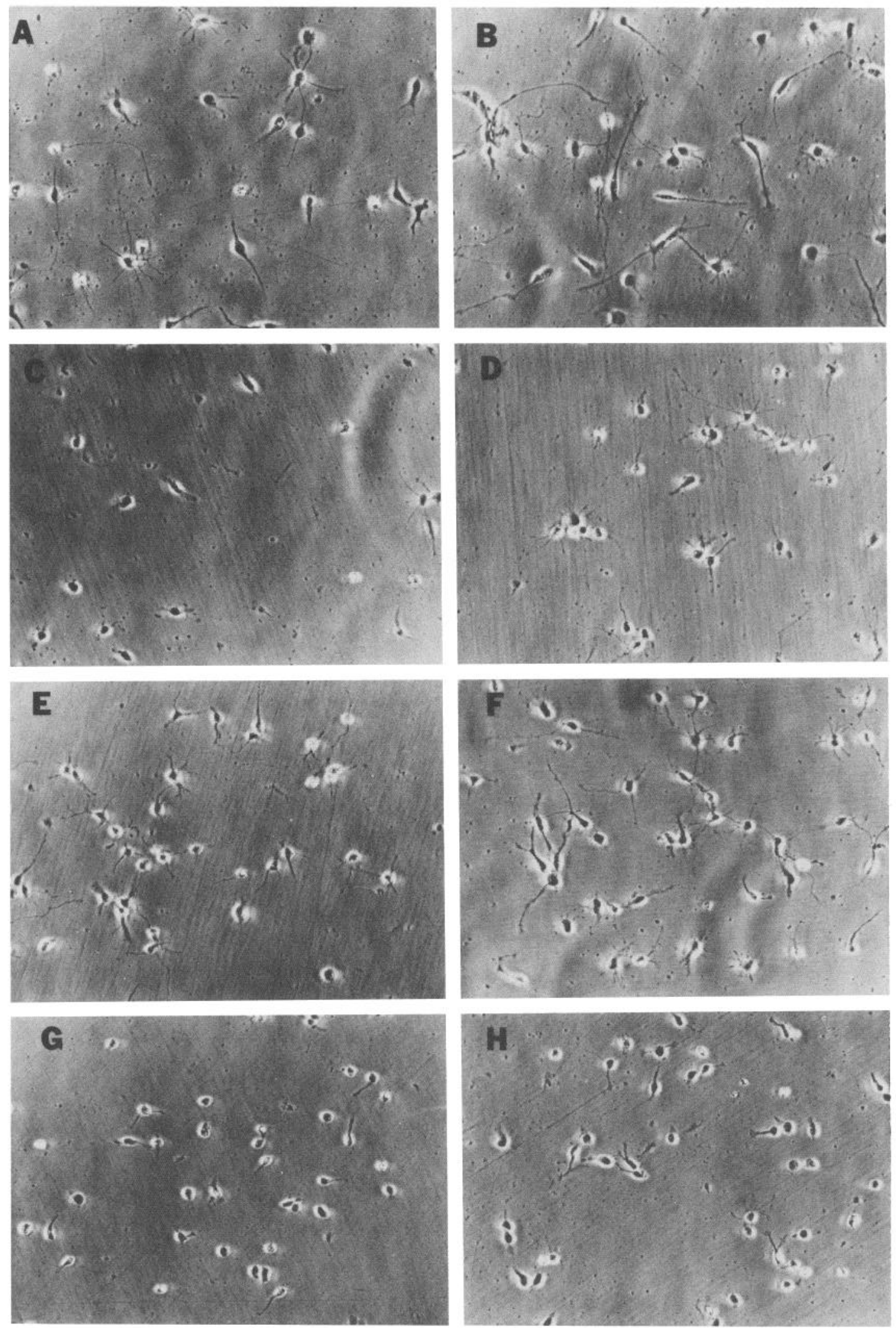

Figure 5. Examples of cultures used to determine process lengths. $A$, hippocampus control. $B$, Hippocampus + bFGF. $C$, EC control. $D$, EC + bFGF. $E$, PC control. $F, \mathrm{PC}+\mathrm{bFGF}$. $G$, St control. $H, \mathrm{St}+\mathrm{bFGF}$. Magnification, $200 \times$. 

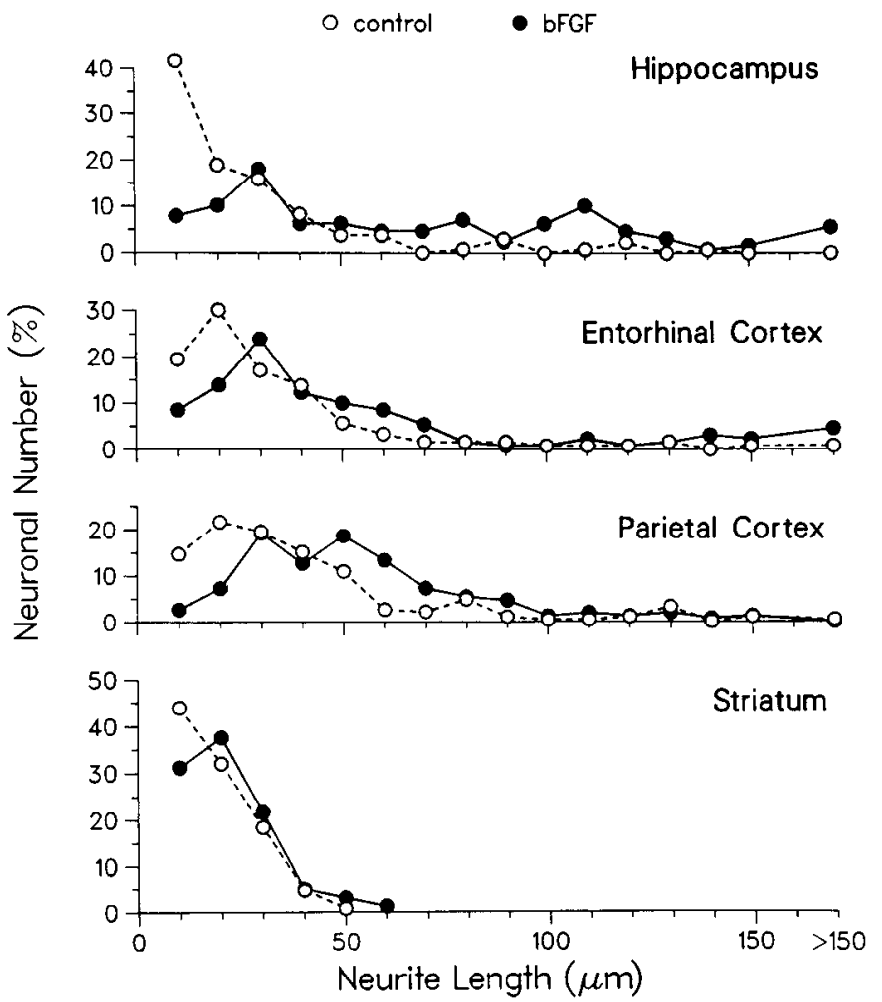

Figure 6. Neurite outgrowth in response to bFGF. Cultures from the indicated regions were grown for $24 \mathrm{hr}$ in the presence of laminin and HDL with or without $1 \mathrm{ng} / \mathrm{ml} \mathrm{bFGF}$. The central region of each of 4 duplicate cultures was photographed (see examples in Fig. 5) and used for process length measurements. Sample size varied from 123 to 184 neurons. Data are presented as percentages for easier comparison. Each point designates the percentage of neurons with a longest process falling within that $10 \mu \mathrm{m}$ bin.

al., 1987). However, HDL decreased the adhesivity of the cells, so that only a highly adhesive surface such as polyornithinelaminin could be employed. Laminin has been demonstrated to be an important component of the extracellular matrix required for neurite outgrowth in studies from several laboratories (Edgar et al., 1984; Lander et al., 1985). From the survey, it appcared that neurons growing on laminin in the presence of HDL demonstrated the best overall neurite outgrowth; any response seen to bFGF in the presence of these other agents that increase neurite outgrowth appeared more likely to be biologically relevant. Inclusion of bFGF still increased neuronal survival under these slightly different culture conditions, but glial proliferation was increased by inclusion of HDL (data not shown).

Photographs of 24-hr-old cultures grown in the absence or presence of bFGF $(1 \mathrm{ng} / \mathrm{ml})$ are shown in Figure 5 . The neurons examined were from the hippocampus, EC, PC, and St. Neurons from the $\mathrm{Sb}$ did not appear to have increased outgrowth in the presence of bFGF (not shown). The longest process of each neuron in a field from the center of each of 4 duplicate cultures was measured; sample size ranged from 123 to 184 neurons. Distribution of process lengths is presented in Figure 6, mean process lengths in Table 3.

Hippocampal neurons showed the greatest response, with mean process length increased nearly 3 -fold and a highly significant shift in the distribution of process lengths $(p<0.001$; chisquare). Neurons from the EC and PC also responded with significant shifts in process length distribution $(p<0.01$, chisquare for EC; $p<0.001$, chi-square for $\mathrm{PC}$ ). For the hippo-
Table 3. Mean process lengths

\begin{tabular}{lll} 
& Control & + bFGF $(1 \mathrm{ng} / \mathrm{ml})$ \\
\hline Hippocampus & $21.5 \pm 2.4$ & $62.6 \pm 4.1^{*}$ \\
Entorhinal cortex & $29.3 \pm 2.8$ & $49.3 \pm 4.0^{*}$ \\
Parietal cortex & $34.4 \pm 2.3$ & $49.0 \mp 2.3^{*}$ \\
Striatum & $12.7 \pm 0.9$ & $15.8 \pm 0.9^{* *}$
\end{tabular}

Neurite outgrowth in the presence of bFGF. Mean process lengths for the cultures illustrated in Figures 5 and 6.

${ }^{*} p<0.01$ versus control.

** $p<0.05$ (Student's $t$ test).

campus and EC, bFGF produced an overall shift to the right in the distribution of process lengths, with noticeable changes in the proportion of neurons having processes longer than $100 \mu \mathrm{m}$. For hippocampal neurons, this increased from 3.8 to $26.0 \%$; for $\mathrm{EC}$, from 4.8 to $14.6 \%$. By contrast, there was relatively little change in this portion of the distribution for the PC neurons: $6.5 \%$ in controls versus $7.3 \%$ with bFGF. Instead, bFGF caused a decrease in neurons with very short processes (of less than 20 $\mu \mathrm{m})$ and an increase in neurons with medium process lengths $(40-60 \mu \mathrm{m})$.

Striatal neurons produced much shorter neurites than did the hippocampal or cortical neurons. Addition of bFGF caused a slight but not significant shift in the distribution of process lengths (Fig. 6D). Even after $6 \mathrm{~d}$ exposure to bFGF, striatal neurons continued to demonstrate quite poor process outgrowth (Fig. 3E).

\section{aFGF increases survival of neurons from multiple regions of the CNS}

In addition to bFGF, brain tissue contains an aFGF. The 2 FGFs are closely related, sharing a total of $53 \%$ sequence homology; however, they are the products of separate and distinct genes (Esch et al., 1985a, b; Gimenez-Gallego et al., 1985; Mergia et al., 1986). aFGF has activity as a mitogen for cells responsive to bFGF, but typically is 10-100-fold less potent (Esch et al., 1985a; Baird et al., 1986; Gospodarowicz et al., 1986; Lobb et al., 1986). It also has previously been demonstrated to have trophic effects on hippocampal neurons in vitro (Walicke and Baird, 1987a). The survival of neurons from 6 regions of the brain was compared in the presence of $1 \mathrm{ng} / \mathrm{ml} \mathrm{bFGF}$ and $10 \mathrm{ng} / \mathrm{ml}$ aFGF (Fig. 7).

The numbers of neurons surviving after $24 \mathrm{hr}$ in vitro in control cultures or with bFGF or aFGF are shown in the upper half of Figure 7. There was no significant difference in survival attributable either to bFGF or aFGF. Survival after $6 \mathrm{~d}$ in vitro is shown in the lower half of Figure 7. For hippocampal neurons, both bFGF and aFGF significantly increased neuronal survival ( $p<0.01$; ANOVA), but aFGF supported fewer neurons. The effects of $b F G F$ on neuronal survival from the remaining regions were consistent with results previously illustrated in Figure 2, with all regions showing significant increases except for the $\mathrm{Sb}$. aFGF significantly increased survival of neurons from the EC, $\mathrm{PC}$, and St. Since the number of neurons supported by aFGF was smaller, the difference did not achieve the same level of significance $(p<0.05$ for each neuronal type; ANOVA). The number of neurons surviving in the presence of aFGF and $b F G F$ differed significantly in each of these 3 regions, at either $p<$ 0.05 or $p<0.01$ (ANOVA).

Although aFGF did somewhat increase the number of neurons present in septal cultures, the difference did not achieve 


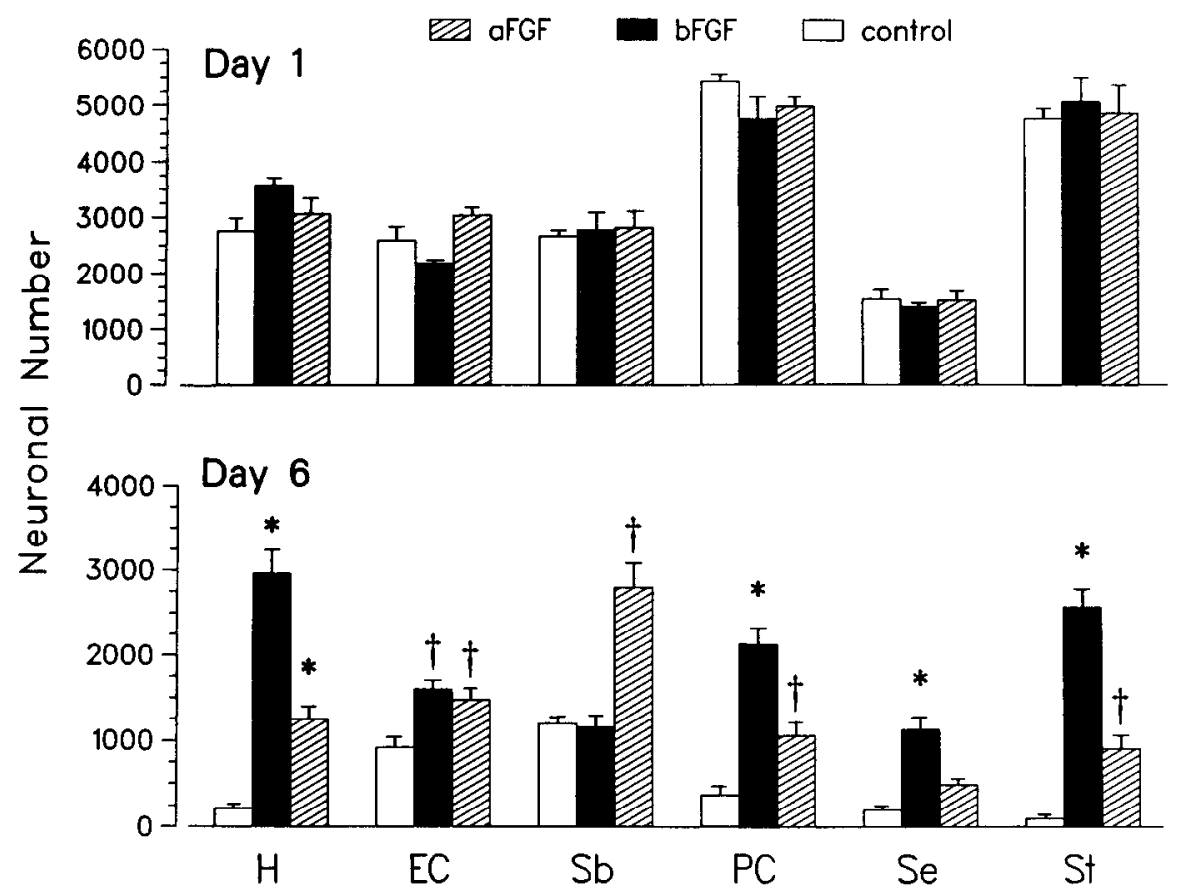

Figure 7. Survival of neurons in the presence of bFGF or aFGF. Abbreviations, as in Figure 1. Bars are mean \pm SEM for 6 duplicate cultures. bFGF was $1 \mathrm{ng} / \mathrm{mll} ;$ aFGF was $10 \mathrm{ng} / \mathrm{ml} .{ }^{*} p<0.01$ versus control. $\dagger p<0.05$ versus control (ANOVA).

significance. As previously observed, bFGF had little influence on survival of neurons from the $\mathrm{Sb}$. Considering the generally lower effectiveness of aFGF, it was surprising that neuronal survival from this region was significantly increased by aFGF from 1200 to 2800 ( $p<0.05$; ANOVA).

Titration curves were performed to determine the concentration of aFGF required to support survival of neurons from the hippocampus, EC, PC, and Sb (Fig. 8). The titration curves obtained with hippocampal neurons, and, to a lesser extent, with EC and PC neurons, had a hump on the rising phasc, possibly suggesting the existence of subpopulations with somewhat different aFGF requirements in these cultures. The somewhat unusual shape of the curves obtained complicated calculation of the half-maximal concentrations required for survival, but for all neuronal types it appears to lie between 2 and $5 \mathrm{ng} / \mathrm{ml}(130$ $330 \mathrm{pM}$ ). Sb neurons appeared to require similar concentrations of aFGF, although they differed from the other 3 populations in their failure to respond to bFGF. Comparing the concentrations of aFGF determined in Figure 8 to those of bFGF determined in Figure 4 suggests that neuronal survival requires at least 100 -fold more aFGF than bFGF, which is consistent with comparisons of potency on other non-neuronal cell types (Esch et al., 1985a; Lobb et al., 1986).

\section{Discussion}

The major finding in these studies is that bFGF can support the survival of neurons from many regions of the CNS. These include neurons from the hippocampus, entorhinal cortex, frontal cortex, parietal cortex, occipital cortex, anterior thalamus, striatum, and septum. bFGF was further demonstrated to stimulate neurite outgrowth from neurons derived from hippocampus, $\mathrm{EC}$, and PC. Since the proportion of glial cells contaminating the cultures from these different regions was less than $2 \%$, it appears likely that bFGF acted directly on the neurons (Walicke and Baird, 1987b). Therefore, bFGF appears to be a trophic factor for neurons from many regions of the CNS.

aFGF also significantly increased the survival of neurons from the hippocampus, entorhinal cortex, subiculum, parietal cortex, and striatum. The concentrations required were considerably higher than for $\mathrm{bFGF}$, and the total number of neurons supported was generally lower. Previous studies with hippocampal neurons demonstrated that astrocytic proliferation under these culture conditions was stimulated less by aFGF than by bFGF (Walicke and Baird 1987b). Although glial content was not formally determined for cultures from all these brain regions, there were no obvious increases in cells of astrocytic morphology in

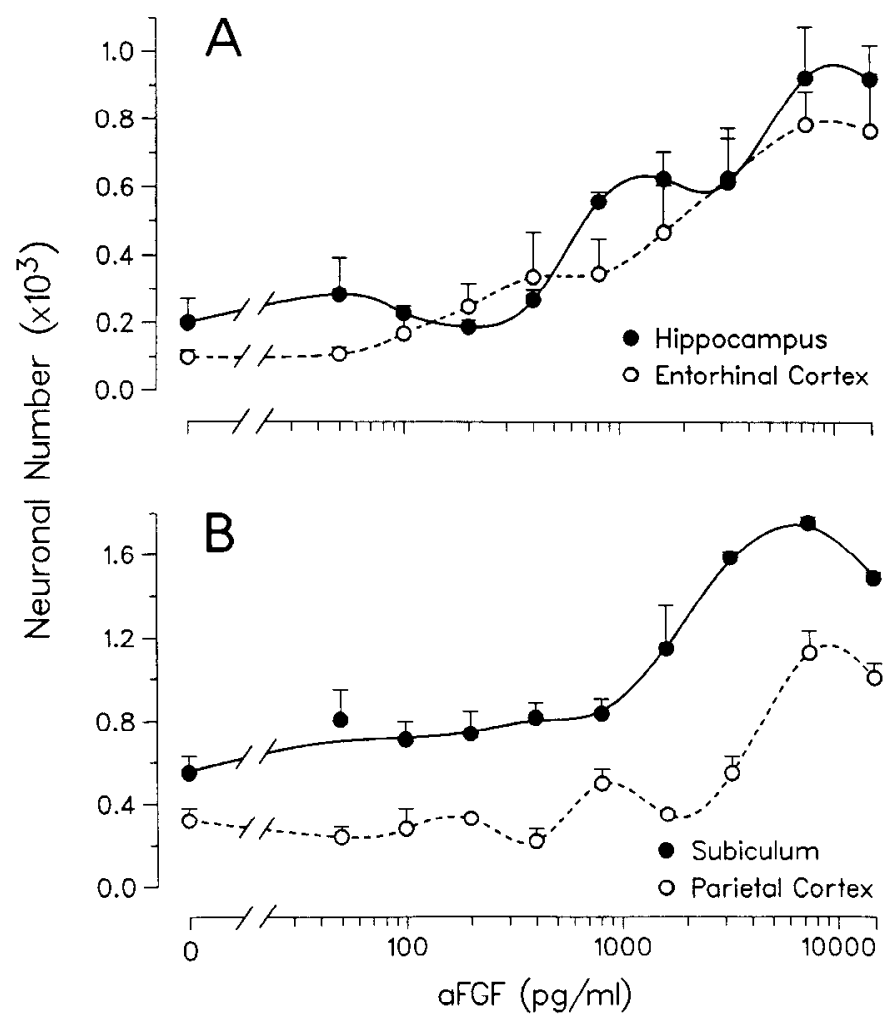

Figure 8. Concentration of aFGF required for neuronal survival. Surviving neurons in 6-d-old cultures were determined for $(A)$ hippocampus and EC; $(B)$ Sb and PC. Points are mean \pm SEM for 3 duplicate cultures. 
phase-microscopic observations. Therefore, aFGF also appears to be a neurotrophic factor for neurons from many regions of the CNS.

Further characterization of the responsive neurons by transmitter phenotype and synaptic targets will be essential for elucidating the roles of bFGF and aFGF in the brain. Given the widespread distribution of these neurons, if a correlation exists between transmitter phenotype and bFGF sensitivity, then the responsive neurons likely use an abundant neurotransmitter, such as GABA or glutamate. There seems, however, to be no $a$ priori reason to assume a common, shared transmitter phenotype since the one well-characterized neurotrophic factor, NGF, addresses adrenergic, cholinergic, and peptidergic neurons (Thoenen and Edgar, 1985).

Despite the limited characterization of the neuronal populations, some inferences can be made about the identity of the responsive neurons. Since the hippocampus was removed from fetuses before the generation of the dentate gyrus, the majority of neurons present in vitro can be reasonably identified as pyramidal neurons (Banker and Cowan, 1979). The neurons surviving in the various cortical cultures resembled the hippocampal neurons, and therefore likely include pyramidal neurons from these regions. Over $90 \%$ of striatal neurons are small- to medium-sized, in contrast to the small subpopulation of large cholinergic neurons (Fentress et al., 1981). Since survival in the presence of bFGF exceeds $10 \%$ of the cells originally placed in culture, small neurons must be responding. This is consistent with the different appearance of striatal cultures from cultures of other brain regions.

Although there was significantly increased survival of neurons from multiple CNS regions, the proportion of neurons supported by bFGF and aFGF varied considerably. The greatest response was observed with the hippocampus, where bFGF appeared to be required by $60 \%$ of the total population. Since there are many rcasons that ncurons might fail to survive in vitro, this may represent a lower limit on the proportion of responsive cells. In other regions, such as the septum and PC, bFGF rescued significantly fewer neurons. Additionally, PC neurons were observed to have a qualitatively different pattern of enhancement in neurite growth than hippocampal or EC neurons. These observations might suggest the existence of a specific subpopulation of bFGF-responsive neurons among the varied neuronal types in the cortex.

Striatal neurons showed several differences in their responses to bFGF from hippocampal and cortical neurons. The minimal quantity of bFGF required for survival was significantly higher. Since the number of neurons supported by supramaximal amounts of bFGF was comparable among these regions, it seems unlikely that the difference merely reflects the poor condition of the striatal cells. Furthermore, the striatal neurons failed to show increased neurite extension in the presence of bFGF. These observations could suggest that bFGF plays a somewhat different role in the development of the basal ganglia than in the cortex.

The most distinctive pattern was observed with neurons derived from the $\mathrm{Sb}$. These neurons did not demonstrate increased survival in the presence of $b F G F$. Under control conditions, they survived, if anything, a bit better than neurons from other regions, suggesting that the poor condition of the cells was not responsible for their lack of response. Furthermore, there was a small but significant increase in their survival in the presence of aFGF. These observations suggest that not all CNS neurons respond to $\mathrm{bFGF}$, though the possibility that $\mathrm{bFGF}$ might be produced by the cells in culture was not excluded. Although $\mathrm{aFGF}$ and bFGF appear to have essentially identical effects on mesenchymal cells (Baird et al., 1986; Gospodarowicz et al., 1986; Lobb et al., 1986), we have previously observed additive effects of bFGF and aFGF on the survival of hippocampal neurons for 1 month in vitro (Walicke and Baird, 1987a). These observations with neurons from the $\mathrm{Sb}$ further support the possibility that aFGF may have some biological effects distinct from those of bFGF in the CNS.

The somewhat different responses of neurons from these various brain regions may reflect distinct biological roles for the FGFs in various portions of the brain. It must be noted, however, that they may also reflect subtle variations among the various types of neuronal cultures. The system employed, though slightly modified from a widely used serum-free medium formulation (Bottenstein and Sato, 1979), lacks a variety of essential vitamins, minerals, lipids, and hormones. Different populations of CNS neurons may have distinct sensitivities to these deficiencies, and may differ in their adaptation to the general culture environment. Astrocyte content was eliminated as a significant variable among the different regions examined; however, interactions among the neurons themselves might also influence survival. For example, there is evidence to suggest that some neuropeptides may have trophic activity (Swaab and Boer, 1983; Payan, 1985; Brenneman and Eiden, 1986). Possibly, endogenous neuronal contents of peptides or even trophic factors could partly account for the regional variations.

These studies suggest that aFGF and bFGF are important trophic factors for neurons from many regions of the CNS. The broad spectrum of responsive neurons suggests a wider range of neuronal specificity than has been previously observed for NGF, and may appear to contradict some predicted models of neurotrophic factor specificity. It should be noted that not all neurons from any of these regions appear to respond to either bFGF or aFGF, and that the data appear to suggest that only subpopulations of cells are responsive in some regions, such as the PC. Since all of these studies employed neurons from E18 rats, the possibility that responsiveness to the FGFs reflects a common developmental stage cannot be excluded.

In addition to their neurotrophic effects, bFGF and aFGF are also mitogens for astrocytes (Morrison and de Vellis, 1981; Pettmann et al., 1985; Walicke and Baird, 1987b) and oligodendrocytes (Eccelston and Silberberg, 1985; Saneto and de Vellis, 1985). Furthermore, they are mitogens for endothelial cells in vitro and have potent angiogenic activity in vivo (Baird et al., 1986; Gospodarowicz et al., 1986; Lobb et al., 1986). The ability of a single factor to stimulate growth of multiple different cell populations in brain tissue suggests the possibility for competitive interactions or coordinated growth regulation, depending on the circumstances. With their multiplicity of activities, it seems likely that aFGF and bFGF play a significant role in the development of the CNS.

\section{References}

Baird, A., F. Esch, P. Mormede, N. Ueno, N. Ling, P. Bohlen, S.-Y. Ying, W. B. Wchrenberg, and R. Guillemin (1986) Molccular characterization of fibroblast growth factor: Distribution and biological activities in various tissues. Recent Prog. Horm. Res. 42: 143-205.

Banker, G. A. (1980) Trophic interactions between astroglial cells and hippocampal neurons in culture. Science 209: 809-810.

Banker, G. A., and W. M. Cowan (1979) Further observations on 
hippocampal neurons in dispersed cell culture. J. Comp. Neurol. 187. 469-494.

Barbin, G., I. Selak, M. Manthorpe, and S. Varon (1984) Use of central neuronal cultures for the detection of neuronotrophic agents. Neuroscience $12: 33-43$.

Bottenstein, J. E., and G. H. Sato (1979) Growth of a rat neuroblastoma cell line in serum-free supplemented medium. Proc. Natl. Acad. Sci. USA 76: 514-519.

Brenneman, D. E., and L. E. Eiden (1986) Vasoactive intestinal peptide and electrical activity influence neuronal survival. Proc. Natl. Acad. Sci. USA 83: 1159-1162.

Eccelston, P. A., and D. H. Silberberg (1985) Fibroblast growth factor is a mitogen for oligodendrocytes in vitro. Dev. Brain Res. 21: 315318.

Edgar, D., R. Timpl, and H. Thoenen (1984) The heparin-binding domain of laminin is responsible for its effects on neurite outgrowth and neuronal survival. EMBO J. 3: 1463-1468.

Esch, F., A. Baird, N. Ling, N. Ueno, F. Hill, L. Denoroy, R. Klepper, D. Gospodarowicz, P. Bohlen, and R. Guillemin (1985a) Primary structure of bovine pituitary basic fibroblast growth factor (FGF) and comparison with the amino-terminal sequence of bovine brain acidic FGF. Proc. Natl. Acad. Sci. USA 82: 6507-6511.

Esch, F., N. Ueno, A. Baird, F. Hill, L. Denoroy, N. Ling, D. Gospodarowicz, and R. Guillemin (1985b) Primary structure of bovine brain acidic fibroblast growth factor (FGF). Biochem. Biophys. Res. Commun. 133: 554-562.

Fentress, J. C., B. B. Stanfield, and W. M. Cowan (1981) Observations on the development of the striatum in mice and rats. Anat. Embryol. 163: 275-298.

Gimenez-Gallego, G., J. Rodkey, C. Bennet, M. Rios-Candelore, J. DiSalvo, and K. Thomas (1985) Brain-derived acidic fibroblast growth factor: Complete amino acid sequence and homologies. Science 230: $1385-1388$.

Gloor, S., K. Odink, J. Guenther, H. Nick, and D. Monard (1986) A glia-derived neurite promoting factor with protease inhibitory activity belongs to the protease nexins. Cell 47: 687-693.

Gospodarowicz, D., G. Neufeld, and L. Schweigerer (1986) Fibroblast growth factor. Mol. Cell. Endocrinol. 46: 187-204.

Hefti, F. (1986) Nerve growth factor promotes survival of septal cholincrgic ncurons after fimbrial transections. J. Neurosci. 6: 2155-2162.

Ignatius, M. J., E. M. Shooter, R. E. Pitas, and R. W. Mahley (1987) Lipoprotein uptake by neuronal growth cones in vitro. Science 236: 959-962.

Lander, A. D., D. K. Fujii, and L. F. Reichardt (1985) Laminin is associated with the "neurite-promoting factors" found in conditioned media. Proc. Natl. Acad. Sci. USA 82: 2183-2187.

Large, T. H., S. C. Bodary, D. O. Clegg, G. Weskamp, U. Otten, and L. Reichardt (1986) Nerve growth factor gene expression in the developing rat brain. Science 235: 352-355.

Lasher, R. S., and I. S. Zagon (1972) The effect of potassium on neuronal differentiation in cultures of dissociated rat cerebellum. Brain Res. 41: 482-488.

Lobb, R. R., J. W. Harper, and J. W. Fett (1986) Purification of heparin-binding growth factors. Anal. Biochem. 154: 1-14.

Mergia, A., R. Eddy, J. A. Abraham, J. C. Fiddes, and T. B. Shows (1986) The genes for basic and acidic fibroblast growth factors are on different human chromosomes. Biochem. Biophys. Res. Commun. 138: 644-651.
Mobley, W. C., J. L. Rutkowski, G. I. Tennekoon, K. Buchanan, and M. V. Johnston (1985) Choline acetyltransferase activity in striatum of neonatal rats increased by nerve growth factor. Science 229:284 287.

Mobley, W. C., L. Rutkowski, G. I. Tennekoon, J. Gemski, K. Buchanan, and M. V. Johnston (1986) Nerve growth factor increases choline acetyltransferase activity in developing basal forebrain neurons. Mol. Brain Res. 1: 53-62.

Morrison, R. S., and J. de Vcllis (1981) Growth of purificd astrocytcs in a chemically defined medium. Proc. Natl. Acad. Sci. USA 78: 7205 7209.

Morrison, R. S., A. Sharma, J. de Vellis, and R. A. Bradshaw (1986) Basic fibroblast growth factor supports the survival of cerebral cortical neurons in primary culture. Proc. Natl. Acad. Sci. USA 83: 75377541.

Payan, D. G. (1985) Receptor-mediated mitogenic effects of substance P on cultured smooth muscle cells. Biochem. Biophys. Res. Commun. 130: 104-109.

Pettmann, B., M. Weibel, M. Sensenbrenner, and G. Labourdette (1985) Purification of two astroglial growth factors from bovine brain. FEBS 189: 102-108.

Saneto, R. P., and J. de Vellis (1985) Characterization of cultured rat oligodendrocytes proliferating in a scrum-frec, chemically defincd mcdium. Proc. Natl. Acad. Sci. USA 82: 3509-3513.

Seiler, M., and M. E. Schwab (1984) Specific retrograde transport of nerve growth factor (NGF) from neocortex to nucleus basalis in the rat. Brain Res. 300: 33-39.

Selak, I., S. D. Skaper, and S. Varon (1985) Pyruvate participation in the low molecular weight trophic activity for CNS neurons in gliaconditioned media. J. Neurosci. 5: 23-28.

Swaab, D. F., and G. J. Boer (1983) Neuropeptides and brain development: Current perils and future potential. J. Dev. Physiol. 983: 6775 .

Taniuchi, M., J. B. Schweitzer, and E. M. Johnson (1986) Nerve growth receptor molecules in rat brain. Proc. Natl. Acad. Sci. USA 83: $1950-1954$.

Thoenen, H., and D. Edgar (1985) Neurotrophic factors. Science 229: 238-242.

Walicke, P. A., and A. Baird (1987a) Trophic effects of fibroblast growth factor on neural tissue. Prog. Brain Res. (in press).

Walicke, P. A., and A. Baird (1987b) Neurotrophic effects of basic and acidic fibroblast growth factor are not mediated through glial cells. Dev. Brain Res. (in press).

Walicke, P., W. M. Cowan, N. Ueno, A. Baird, and R. Guillemin (1986a) Fibroblast growth factor promotes survival of dissociated hippocampal neurons and enhances neurite extension. Proc. Natl. Acad. Sci. USA 83: 3012-3016.

Walicke, P. A., S. Varon, and M. Manthorpe (1986b) Purification of a human red blood cell protein supporting the survival of cultured CNS neurons, and its identification as catalase. J. Neurosci. 6: 11141121.

Williams, L. R., S. Varon, G. M. Peterson, K. Wictorin, W. Fischer, A. Björklund, and F. H. Gage (1986) Continuous infusion of nerve growth factor prevents basal forebrain neuronal death after fimbria fornix transection. Proc. Natl. Acad. Sci. USA 83: 9231-9235. 